\title{
Comparison of urinary 6- $\beta$-cortisol and the erythromycin breath test as measures of hepatic P450IIIA (CYP3A) activity
}

\begin{abstract}
The production of ${ }^{14} \mathrm{CO}_{2}$ in the breath from an intravenous dose of $\left[{ }^{14} \mathrm{C}-\mathrm{N}\right.$-methyl]-erythromycin (the erythromycin breath test [ERMBT]) and the measurement of the ratio of $6-\beta$-cortisol to free cortisol $(6-\beta-F / F F)$ in the urine have each been proposed as means of measuring hepatic P450IIIA catalytic activity in patients. We found that there was a significant correlation between the results of each test $(r=$ $0.59, p<0.001$ ) in 47 patients who were without liver disease and who were not taking medications believed to influence P450IIIA catalytic activity. In the 24 of these patients who were subsequently treated with the P450IIIA substrate cyclosporine, the ERMBT result was highly correlated with the mean trough cyclosporine blood level observed; however, there was no correlation between urinary 6- $\beta$ F/FF and the cyclosporine blood levels. In a separate study of a patient during the anhepatic phase of liver transplantation surgery, the ERMBT result decreased by greater than $85 \%$, whereas urinary $6-\beta$ F/FF decreased by just $50 \%$. We conclude that the ERMBT and urinary 6- $\beta-F / F F$ do not always provide similar information about P450IIIA catalytic activity in patients, possibly because of extrahepatic production of 6- $\beta-F$. Of the two tests, the ERMBT appears to provide the most relevant information for cyclosporine administration. (Chin Pharmacol Ther 1992;52:265-73.)
\end{abstract}

\section{Paul B. Watkins, $M D,{ }^{a}$ D. Kim Turgeon, MD, Paul Saenger, MD, Kenneth S. Lown, MD, Joseph C. Kolars, MD, Ted Hamilton, MS, Kenneth Fishman, Philip S. Guzelian, MD, and John J. Voorhees, MD Ann Arbor, Mich., New York, N.Y., and Richmond, Va.}

The P450IIIA*1 enzymes catalyze the major pathways for the metabolism of many commonly used medications, including erythromycin, ${ }^{2}$ cyclosporine, ${ }^{3}$ midazolam, ${ }^{4}$ lidocaine,${ }^{5}$ tamoxifen, ${ }^{6}$ and calcium channel blockers. ${ }^{7}$ P450IIIA enzymes have also been implicated in the bioactivation of potentially toxic environmental chemicals such as mycotoxins. ${ }^{8,9}$ There

From the Departments of Medicine and Dermatology, University of Michigan Medical Center, Ann Arbor; the Department of Pediatrics, Montefiore Medical Center/Albert Einstein College of Medicine, New York; and the Department of Medicine, Medical College of Virginia, Richmond.

Sponsored by grant No. 5-MOI-RR00042 from the General Clinical Research Center of the University of Michigan Medical Center (Ann Arbor, Mich.).

Received for publication March 4, 1992; accepted May 12, 1992.

Reprint requests: Paul B. Watkins, MD, Room A7119, University of Michigan Medical Center, 1500 East Medical Center Dr., Ann Arbor, MI 48109-0108.

${ }^{\text {a}}$ Supported by grants GM38139 and ES04238 from the National Institutes of Health (Bethesda, Md.).

*In this article, the term "P450IIIA" is used to describe enzymes within the CYP3A gene subfamily. ${ }^{1}$

13/1/39336. exists at least a tenfold interindividual variation in the liver catalytic activity of P450IIIA that may reflect both genetic and environmental factors. ${ }^{10.11}$ It seems likely that this heterogeneity accounts, at least in part, for interpatient differences both in the dosing requirements of some drugs and in the susceptibility to some environmental diseases. To test these hypotheses it would be desirable to have a noninvasive test capable of quantitating P450IIIA activity in patients.

We have proposed that the catalytic activity of P450IIIA enzymes in a patient's liver can be inferred from the rate at which the patient exhales ${ }^{14} \mathrm{CO}_{2}$ after receiving a trace intravenous dose of $\left[{ }^{14} \mathrm{C}-\mathrm{N}\right.$-methyl]erythromycin. ${ }^{12}$ This test is based on the observation that P450IIIA exclusively catalyzes the $N$-demethylation of erythromycin in human liver microsomes ${ }^{12}$ and the fact that the carbon atom in formaldehyde produced by the demethylation should largely appear in the breath as carbon dioxide. ${ }^{13}$ We believe that the erythromycin breath test (ERMBT) selectively measures P450IIIA activity on the basis of several observations. First, the rate of production of breath ${ }^{14} \mathrm{CO}_{2}$ 
increases significantly in patients after they are treated with inducers of P450IIIA and decreases significantly in patients after they are treated with triacetyloleandomycin, a known selective inhibitor of P450IIIA. ${ }^{12}$ Second, the ERMBT result obtained in an individual patient correlates with the trough blood levels of cyclosporine that are observed when the patient receives the drug. ${ }^{14}$ Finally, we have recently performed a study where the ERMBT was administered to nine patients with liver disease who were awaiting liver transplantation. ${ }^{15}$ We found that there was a significant correlation between the preoperative ERMBT result and the level of P450IIIA measured directly in the patient's liver tissue obtained at the time of transplant surgery. In contrast, we found no correlation between the breath test result and the liver content of four other major P450 enzymes.

P450IIIA enzymes have also been shown to catalyze the 6- $\beta$-hydroxylation of steroid hormones. ${ }^{16}$ It has therefore been suggested that measurement of the ratio of $6-\beta$-cortisol to free cortisol $(6-\beta-F / F F)$ in the urine also provides a means of determining hepatic P450IIIA activity in patients. ${ }^{17,18}$ Because urinary $6-\beta-F / F F$ measurements are completely noninvasive and do not involve radioisotopes, this assay would be preferable to the ERMBT in most instances if both assays provided similar information. However, no correlation between $6-\beta-\mathrm{F} / \mathrm{FF}$ and the erythromycin breath test was observed by Hunt et al. ${ }^{19}$ in a small number of patients with hypertension. To investigate the relationship between these breath and urine assays, we administered both tests to 48 patients under a variety of experimental conditions.

\section{METHODS}

Patients studied. A total of 48 patients received both the ERMBT and a 24-hour urine collection for measurement of $6-\beta-F / F F$. Each patient received their ERMBT within 24 hours of completing the urine collection. A single patient had end-stage liver disease and underwent liver transplantation. He is discussed separately below (anhepatic man) and in the Results section. The remaining 47 patients had no history of hepatic or renal dysfunction; chemistries used to assess the integrity of the liver (transaminases, bilirubin, and albumin) and kidney (blood urea nitrogen and creatinine) were within $20 \%$ of the normal range in all patients. Twenty-two of these patients were stable inpatients who were receiving fixed medication regimens. The medications and some characteristics of these patients have been reported previously (patients numbered 1 through $5,7,8,10,11,14$ through 20 ,
22 through $24,25,28,29$, and $30^{12}$ ). The remaining 25 patients were obtained from a study of 33 outpatients with psoriasis who were being treated with cyclosporine in an experimental protocol. These patients were not receiving systemic medications other than cyclosporine and are described elsewhere. ${ }^{14}$ All 33 patients enrolled in this study were asked to collect their urine at home for 24 hours before their visits to the hospital for the ERMBT and other studies. Six patients failed to bring in a urine sample, and in two cases the urine sample was lost or mislabeled. None of the 47 patients was receiving medications known or suspected to induce or inhibit the catalytic activity of P450IIIA (glucocorticoids, antiseizure medications, imidazole, antimycotic drugs, or macrolide antibiotics $^{10,11}$ ).

After the initial urine collections and breath tests, several of the hospitalized patients underwent repeat urine collections and breath tests after they were voluntarily treated with rifampin $(600 \mathrm{mg}$ daily for 4 days) or triacetyloleandomycin (single $250 \mathrm{mg}$ dose), a known inducer and inhibitor, respectively, of P450IIIA. ${ }^{2,17}$ We have shown previously that these treatment regimens result in significant increases and decreases, respectively, in the ERMBT results. ${ }^{12}$ The ERMBT results obtained in these patients have been reported previously. ${ }^{12}$

The erythromycin breath test. The ERMBT was administered and the results were analyzed as previously described. ${ }^{12}$ The results were expressed as the percentage of administered carbon 14 exhaled in the 1 hour after the intravenous injection of $\left[{ }^{14} \mathrm{C}-\mathrm{N}\right.$-methyl]erythromycin. We have shown that there is less than a $20 \%$ intrapatient variability in the ERMBT result (Watkins et al. ${ }^{12}$ and Watkins $\mathrm{P}$, unpublished data, March 1992). In the patients receiving cyclosporine, all breath tests were performed before the medication was started.

Measurement of urinary 6- $\beta-F / F F$. Urine was collected for 24 hours in a single container. The total voided volume was measured, and aliquots were frozen and stored at $-20^{\circ} \mathrm{C}$. Aliquots were batched and sent on ice to the laboratory of Dr. Saenger. $6-\beta$-Cortisol concentration was determined by a radioimmunoassay that has been previously validated. ${ }^{20,21}$ Paired aliquots of urine were also shipped to Hazelton Laboratories, Vienna, Va., where free cortisol concentration was determined by radioimmunoassay. ${ }^{22}$

Blood measurements of cyclosporine. Cyclosporine was measured in whole blood by an HPLC technique. $^{23}$

Studies in an anhepatic man. Urine was collected 


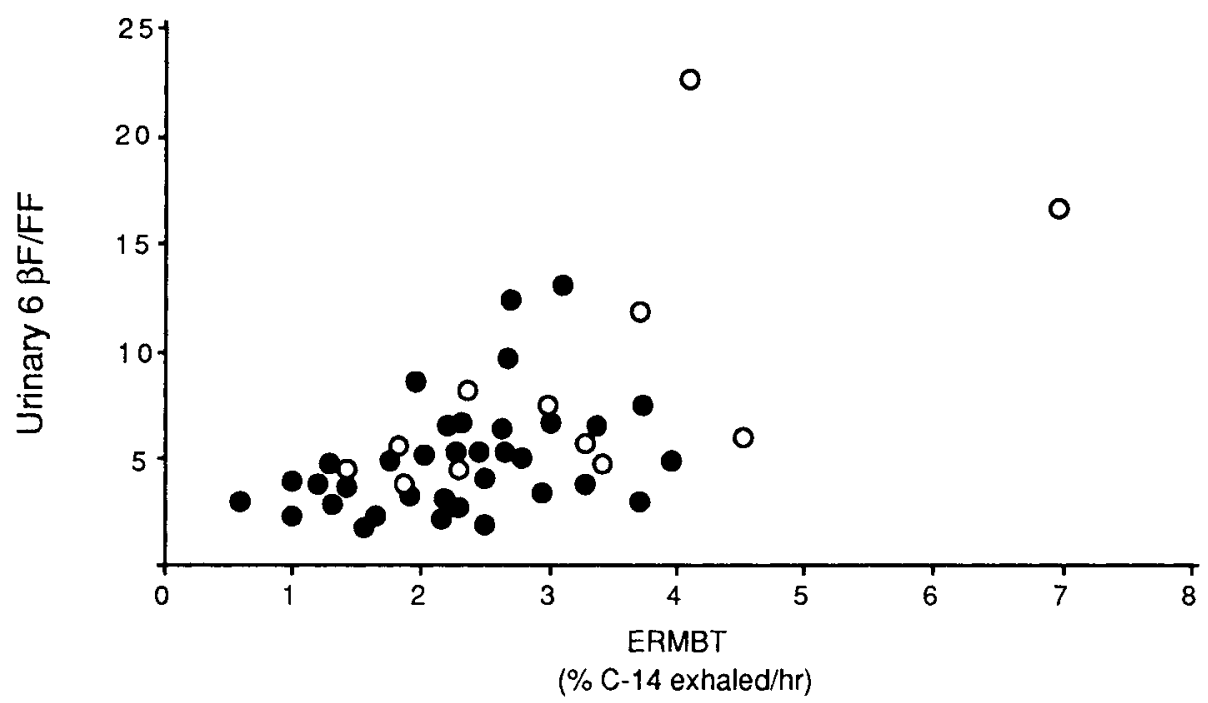

Fig. 1. Correlation between the erythromycin breath test (ERMBT) result and urinary 6- $\beta$ cortisol/free cortisol ratio $(6-\beta-F / F F)$ in 47 patients. Patients collected their urine for 24 hours before receiving the ERMBT. The ERMBT was performed and urine $6-\beta-F / F F$ was assayed as described in the Methods section. Solid circles, men; open circles, women.

from and the ERMBT was administered to a 31-yearold man who underwent liver transplantation for endstage liver disease attributed to chronic non- $\mathrm{A}$, non- $\mathrm{B}$ hepatitis. During surgery, there is a 2- to 3-hour interval in which the patient is anhepatic; that is, the diseased liver is removed from the recipient's body but perfusion to the donor liver has not yet been established. During this interval, blood from the inferior vena cava (which drains the lower extremities and the kidneys) and blood from the portal vein (which drains the intestine) is diverted by tubing to an extracorporeal pump. The pump feeds the blood back through a catheter into the left subclavian vein. In this way, blood perfusion is maintained to all the body tissues.

The ERMBT was administered and consecutive 15minute urine samples were obtained during this patient's anhepatic state. For the breath test, the patient's exhaled breath was sampled by placing a T-tube into the exhalation tubing of the patient's respirator. Urine samples were obtained from an indwelling urinary catheter.

Statistical analysis. The strength of the linear relationship between the ERMBT and $6-\beta-F / F F$ results was assessed with Pearson's product-moment correlation coefficient. Selection of the best set of variables for predicting cyclosporine blood levels was performed with a forward stepwise multiple linear regression analysis whereby potential predictor (independent) variables were sequentially included or excluded from the model according to the strength of their respective partial correlations. The dependent variable was expressed in the common logarithms of the mean cyclosporine blood levels to provide a better linear fit to the model. Rationale for the selection of the independent variables examined has been reported previously. $^{14}$

All $p$ values are two tailed. The data were analyzed with the Michigan Interactive Data Analysis System (MIDAS), a statistical software package developed by the Statistical Research Laboratory at the University of Michigan (Ann Arbor, Mich.).

\section{RESULTS}

ERMBT results and $6-\beta-F / F F$ ratios. Twentyfour-hour urine collections were obtained from and the ERMBT was administered to 47 patients who had no clinical evidence of liver disease. These patients were not receiving medications known or suspected to influence the activity of P450IIIA (see Methods). In Fig. 1, the results of the urine $6-\beta-F / F F$ ratios are compared with the corresponding ERMBT results obtained for each patient. There was a positive correlation between the breath test result and $6-\beta-F$ excretion in urine that was highly statistically significant $(r=$ $0.59, p=0.0001)$. However, when the individual with the highest breath test result $(7 \%)$ and the individual with the highest urinary $6-\beta-F / F^{23}$ were excluded from the analysis, the correlation between the 
two tests was less impressive $(r=0.4, p<0.006)$. No patient with a very low ERMBT result $(<1.5 \%)$ had a high urine $6-\beta-F / F F$ ratio $(>5.0)$. However, some patients with a high ERMBT result $(>3.0 \%)$ had a low urinary $6-\beta-F / F F$ ratio $(<5.0$; Fig. 1$)$.

The mean breath test result was higher in women than in men $(3.14 \%$ and $2.29 \%$, respectively, $p<$ 0.02 . unpaired $t$ test). Mean urine $6-\beta-\mathrm{F} / \mathrm{FF}$ ratios were also higher in women than men $(8.5$ and 5.17, respectively, $p<0.01$, unpaired $t$ test). This gender effect largely accounted for the correlation between the two assays. However, when the 34 men and 13 women were analyzed separately, the correlation between the results of the two tests remained significant $(r=0.37, p=0.03$ and $r=0.64, p=0.03$, respectively).

Effect of treatment with rifampin and triacetyloleandomycin. Immediately after the urine collection and breath testing, four patients received the P450IIIA-inducer rifampin and underwent repeat urine collection and breath testing. Rifampin treatment resulted in increases in both urinary $6-\beta-\mathrm{F} / \mathrm{FF}$ and in erythromycin $N$-demethylation in each patient (Fig. 2, A). However, in one patient (Fig. 2, A, hollow triangles) the rifampin treatment resulted in a nearly threefold increase in urinary $6-\beta-\mathrm{F} / \mathrm{FF}$, whereas the patient's breath test result remained almost unchanged.

An additional four patients received a single dose of the selective P450IIIA inhibitor triacetyloleandomycin. The breath test was readministered 2 hours after the triacetyloleandomycin dose and urine was collected during the subsequent 24 hours. As can be seen in Fig. 2, $B$, triacetyloleandomycin treatment appeared to have discordant effects on the results of each test. There was a marked decrease in erythromycin demethylation in each patient, whereas $6-\beta-F$ excretion actually increased in two patients.

Studies in an anhepatic man. We next studied a 30-year-old man who underwent liver transplantation for severe chronic liver disease. Urine was collected and the ERMBT was administered before the liver transplant procedure and again after surgery when the patient was medically stable. The tests were also administered to the patient intraoperatively during the anhepatic phase of the liver transplant operation (see Methods section).

As can be seen in Fig. 3, $A$, the patient's breath test results were considerably higher after the transplant procedure than before. During the anhepatic phase, production of carbon dioxide from erythromycin decreased dramatically $(>85 \%$ compared with the pre- transplant value and $>90 \%$ compared with the posttransplant value). The ratio of $6-\beta-F / F F$ in the urine did not follow a similar pattern. The urinary $6-\beta-F / F F$ ratio was actually higher in the individual before he underwent liver transplantation than after he had received the healthy donor liver (Fig. 3, B). During the anhepatic phase, the rate of production of free cortisol in the urine and the rate of urine production remained constant (data not shown). As can be seen in Fig. 3, $B$, the ratio of $6-\beta-\mathrm{F} / \mathrm{FF}$, which was measured in sequential 15-minute urine collections, decreased approximately $50 \%$ during the first hour but then appeared to plateau, remaining roughly constant for the final $1 \frac{1}{2}$ hours of the anhepatic state. The marked decrease in the urinary $6-\beta-F / F F$ observed when the donor liver was reperfused (Fig. 3, B, arrow) may be related to the significant blood loss and simultaneous infusion of donor blood that occurred during this period.

6- $\beta$-Cortisol as a predictor of cyclosporine blood levels. The above studies show that the urine $6-\beta-F / F F$ ratio and the ERMBT result do not always give similar results. Because one of the potentially important uses for noninvasive assays of P450IIIA activity may be to guide physicians in correct dosing of drugs that are P450IIIA substrates, we wanted to determine which of the two tests was most useful in this regard. We have reported that the ERMBT significantly correlated with the logarithm of the mean trough blood level of cyclosporine in 32 patients who received the drug as an experimental treatment for psoriasis. ${ }^{14}$ Our sample of 47 patients (Fig. 1) included 25 patients enrolled in this study. Each patient was randomized to receive one of three doses of cyclosporine $(3.0,5.0$, or $7.5 \mathrm{mg} / \mathrm{kg} /$ day) and had monthly trough blood levels of cyclosporine determined in whole blood by HPLC. One patient was documented to have poor compliance with his cyclosporine and was excluded from the analysis in our previous study. ${ }^{14}$ We therefore analyzed the data obtained in the remaining 24 patients.

To compare urinary $6-\beta-\mathrm{F} / \mathrm{FF}$ measurements with the ERMBT as a predictor of mean trough cyclosporine blood levels, we performed a multiple regression analysis with the logarithm of the mean cyclosporine blood level as the dependent variable and cyclosporine dose, urinary $6-\beta-F / F F$ ratio, ERMBT result, patient age, and other patient characteristics or laboratory parameters as the independent variables (Table I). In the first step of the regression (Table I, step 1), cyclosporine dose, ERMBT, and patient age were the only variables that significantly correlated with the cyclospor- 



Fig. 2. Changes in the results of the ERMBT (left) and the urinary 6- $\beta-F / F F$ (right) in patients treated with $600 \mathrm{mg} /$ day rifampin for 4 days $(\mathbf{A})$ and a single oral dose of $250 \mathrm{mg}$ triacetyloleandomycin (B). Patients received both tests before and after the indicated treatment. Lines connect the results obtained for each individual.

ine blood levels $(p<0.05)$. The cyclosporine dose was superior to the other variables as a predictor of drug blood level and was incorporated into the model in the first regression step. After interpatient differences in dose of cyclosporine received were thus taken into account, the ERMBT result and patient age were the only significant predictors of cyclosporine trough blood levels (Table I, step 1). Urinary 6- $\beta$ $\mathrm{F} / \mathrm{FF}$ did not appear to have any predictive value in the model before or after the ERMBT values were incorporated into the model (Table I, steps 1 and 2, respectively).

\section{DISCUSSION}

The primary goal of our study was to determine whether the ERMBT and urine 6- $\beta-F / F F$ yielded comparable information. These tests were comparable in that $6-\beta-F / F F$ ratio measured in the urine correlated significantly with the ERMBT test result in the initial 47 patients studied (Fig. 1). Moreover, the mean of the results for both tests was higher in women than in men, which is consistent with earlier reports. ${ }^{12.24}$ In addition, we found that both the urine $6-\beta-F / F F$ and the ERMBT result increased in patients after they were treated with the P450IIIA inducer rifampin, con- 

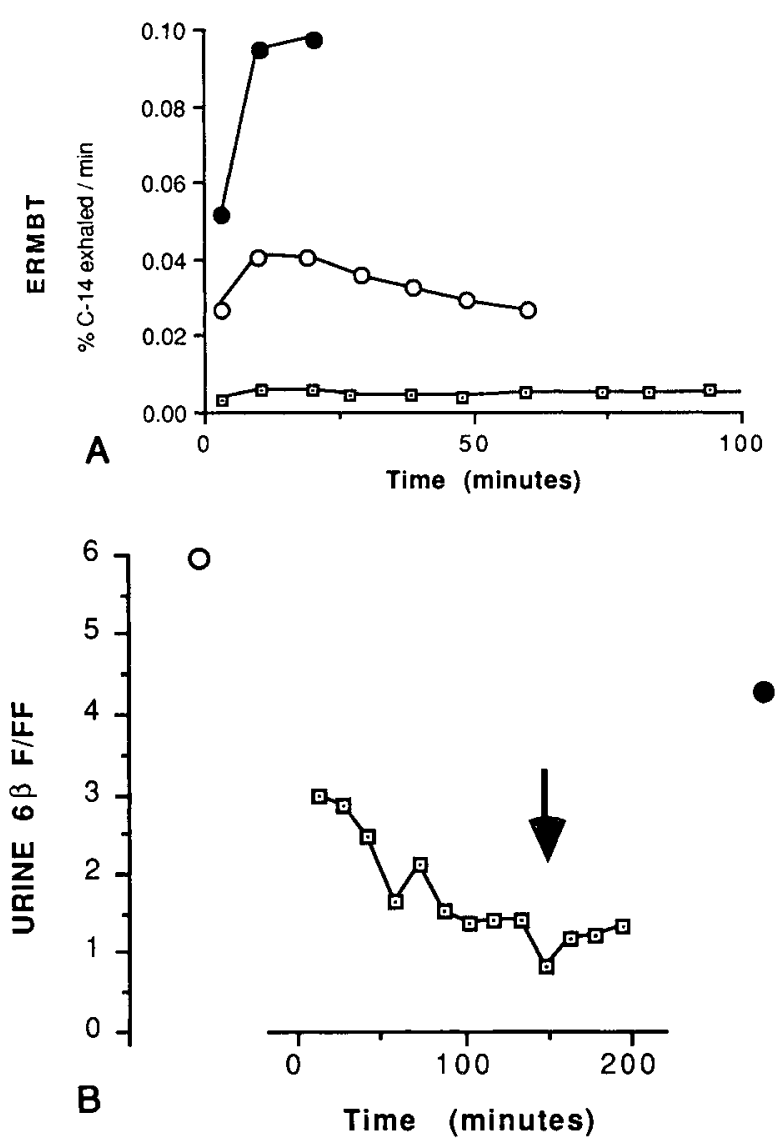

Fig. 3. Changes in the rate of production of carbon dioxide $\left(\mathrm{CO}_{2}\right)$ from erythromycin $(\mathrm{A})$ and in urinary $6-\beta-F / F F(B)$ in a patient undergoing liver transplantation for severe endstage liver disease. The patient underwent testing before (open circles) and after (solid circles) the liver transplantation procedure. In addition, the patient received an intravenous injection of $\left[{ }^{14} \mathrm{C}-\mathrm{N}\right.$-methyl $]$-erythromycin and had sequential 15-minute urine collections for $6-\beta-F / F F$ measurements during the anhepatic phase of the liver transplant procedure (boxes). The rate of $\mathrm{CO}_{2}$ production from erythromycin was estimated from the specific activity of $\mathrm{CO}_{2}$ in the breath sample collected at each time point and an estimation of the endogenous rate of production of $\mathrm{CO}_{2}{ }^{12}$ The arrow (B) indicates the end of the anhepatic phase (when the donor liver was connected to the recipient's blood circulation)

firming earlier observations. ${ }^{12.17}$ Finally, the results of both tests decreased significantly during the anhepatic state, suggesting that hepatic P450IIIA enzymes were largely being measured by both tests.

Nonetheless, striking differences between the results of the two tests were observed in some instances.
Some patients with relatively high breath test results had relatively low urinary $6-\beta-F / F F$ ratios (Fig. 1). In one patient, rifampin treatment resulted in a large relative increase in urine $6-\beta-\mathrm{F} / \mathrm{FF}$ but a minimal change in the breath test result (Fig. 2, A). In addition, whereas a single dose of triacetyloleandomycin dramatically inhibited erythromycin demethylation in each of the four patients studied, this treatment resulted in an increase in the urinary $6-\beta-F / F F$ ratio in two of them (Fig. 2, B). It should be remembered that the urinary measurements reflect the mean production of $6-\beta-F$ over 24 hours, whereas erythromycin demethylation was determined over 1 hour ( 2 hours after administration of triacetyloleandomycin). This may be important because triacetyloleandomycin appears to induce P450IIIA and to inhibit the enzyme. ${ }^{2}$ It is therefore possible that transient inhibition of P450IIIA activity by triacetyloleandomycin followed by induction of P450IIIA catalytic activity could explain the discrepancy between the ERMBT and $6-\beta-F / F F$ results.

There was also a marked difference between the results of each test performed before and after our patient underwent liver transplantation. Replacement of our recipient's diseased liver with a healthy donor liver resulted in a marked increase in the ability of the patient to demethylate erythromycin as we have observed in nine other patients undergoing liver transplantation. ${ }^{15}$ In contrast, liver transplantation resulted in a decrease in urinary $6-\beta-\mathrm{F} / \mathrm{FF}$ (Fig. $3, B$ ).

It is not clear from our data why there is a disparity between the urine and breath test results. It is unlikely that this reflects a problem with the collection of urine because $6-\beta-F / F F$ does not appear to show much diurnal variation; therefore a complete 24-hour collection should be unnecessary (Bienvenu et al. ${ }^{18}$ Saenger, ${ }^{20}$ and Saenger $\mathrm{P}$, unpublished observations, March 1992). Some clues to the basis for the discrepancy may be provided by our studies during the anhepatic state. There was a dramatic reduction in the rate of $\mathrm{CO}_{2}$ production from erythromycin during the anhepatic state (Fig. 3, A). This is consistent with our assumption that the major site of metabolism of the intravenously administered dose of erythromycin would be the liver. However, P450IIIA enzymes are present in some extrahepatic tissues where they may catalyze significant metabolism. Human jejunal mucosa and kidney contain protein and messenger ribonucleic acid closely related to the P450IIIA family of enzymes. ${ }^{25,26}$ Orally administered substrates for P450IIIA appear to undergo significant first-pass metabolism by P450IIIA in the gut, ${ }^{27.28}$ but it is not 
Table I. Summary of the stepwise selection procedure used in creating a multiple regression model capable of predicting the logarithm of the observed mean cyclosporine blood level

\begin{tabular}{|c|c|c|c|c|}
\hline Step & Variable* & $R+$ & p Value & $R^{2} \ddagger$ \\
\hline \multirow[t]{11}{*}{1} & Dose & 0.66 & 0.005 & \multirow[t]{11}{*}{0.43} \\
\hline & ERMBT & -0.70 & 0.0002 & \\
\hline & Age & 0.50 & 0.016 & \\
\hline & HDL cholesterol & -0.39 & 0.065 & \\
\hline & Weight & 0.35 & 0.102 & \\
\hline & Gender & -0.32 & 0.138 & \\
\hline & Triglycerides & 0.26 & 0.228 & \\
\hline & $6-\beta-F / F F$ & -0.24 & 0.280 & \\
\hline & LDL cholesterol & -0.17 & 0.446 & \\
\hline & Total cholesterol & -0.17 & 0.446 & \\
\hline & Body surface area & 0.12 & 0.575 & \\
\hline \multirow[t]{10}{*}{2} & $E R M B T$ & -0.70 & 0.0002 & \multirow[t]{10}{*}{0.71} \\
\hline & Age & 0.46 & 0.032 & \\
\hline & Weight & 0.27 & 0.221 & \\
\hline & Triglycerides & 0.25 & 0.253 & \\
\hline & Body surface area & 0.20 & 0.363 & \\
\hline & Gender & -0.13 & 0.572 & \\
\hline & LDL cholesterol & -0.12 & 0.590 & \\
\hline & HDL cholesterol & -0.05 & 0.833 & \\
\hline & $6-\beta-F / F F$ & -0.02 & 0.913 & \\
\hline & Total cholesterol & -0.02 & 0.932 & \\
\hline
\end{tabular}

ERMBT, Erythromycin breath test; HDL, high-density lipoprotein; $6-\beta-F / F F$, ratio of $6-\beta$-cortisol to free cortisol in urine: $L D L$, low-density lipoprotein

* Variables listed at each regression step are in rank order of predictive capacity. The variables in italics were selected for incorporation into the model at that step. tPartial correlation cocfficient.

+Coefficient of multiple determinations reflecting the predictive capacity of the model after incorporation of the variable selected at that step (italicized). At step 2. the coefficient $(0.71)$ reflects the predictive capacity of the model incorporating both the dose and ERMBT terms.

known whether intravenously administered substrates undergo significant intestinal metabolism. Likewise, the contribution that renal P450IIIA enzymes make to metabolism of P450IIIA substrates is not known. If there is significant extrahepatic metabolism of the intravenously administered erythromycin, it could have been missed in our anhepatic phase study if general anesthesia or the removal of the liver dramatically altered one carbon pool kinetics. The former is unlikely because a dramatic decrease in the ERMBT result was not seen when we administered the ERMBT to a patient with an intact liver while he was receiving general anesthesia (unpublished observations, March 1992). The latter possibility cannot be excluded because the contribution of extrahepatic enzymes to one carbon pool kinetics is not known. However, our previous direct demonstration of the correlation between the ERMBT result and liver levels of P450IIIA ${ }^{15}$ supports the idea that the ERMBT mainly reflects hepatic P450IIIA activity.

The rate of $6-\beta-F$ elimination also decreased sharply during the first hour of the anhepatic state. The observed rate of decline is in good agreement with the serum half-life of $6-\beta-F$ of 48 minutes $^{29}$ and supports a hepatic component to the metabolism measured by the urine test. However, our data are inconsistent with the assumption that the urinary $6-\beta-\mathrm{F} / \mathrm{FF}$ ratio mainly reflects hepatic metabolism. After the first hour of the anhepatic state, the rate of $6-\beta-F$ secretion remained at roughly $50 \%$ of the original value until the donor liver was perfused (Fig. 3, B). Moreover, the $6-\beta-\mathrm{F} / \mathrm{FF}$ ratio was quite high when the patient had end-stage liver disease (before surgery) and actually decreased after he received a new liver. These observations strongly suggest that a component of the $6-\beta-F$ measured in urine is derived from extrahepatic sources. One likely source for extrahepatic production of $6-\beta-F$ is the kidney. Greater amounts of $6-\beta-F$ are found in urine than can be accounted for by simple plasma filtration ${ }^{30}$ and human kidney tissue slices are capable of forming the 6- $\beta$-hydroxy metabolite from cortisol. ${ }^{31}$ The adrenal gland also appears to be capable of producing $6-\beta-\mathrm{F}^{30}$

Our suggestion that urinary $6-\beta-F$ does not always provide an accurate assessment of hepatic P450IIIA activity is consistent with data presented by Ged et al. ${ }^{17}$ These investigators measured urinary $6-\beta-F / F F$ and performed liver biopsies on patients before and af- 
ter they were treated with rifampin. They found that liver P450IIA levels and urine 6- $\beta$-F/FF each increased when patients were treated for several days with this P450IIIA inducer. When results obtained in these patients both before and after treatment were considered in aggregate, there was a significant correlation between $6-\beta-F / F F$ and liver P450IIIA levels. However, if the results obtained in patients before or after treatment with rifampin are analyzed separately, there was no correlation at all between urine $6-\beta-F / F F$ and liver P450IIIA levels. ${ }^{17}$

Regardless of the relative contribution that hepatic and extrahepatic enzymes make to the results of each test, a clinically relevant question is which test provides the most useful information regarding individualized dosing of administered P450IIIA substrates. In this regard, the ERMBT was clearly superior as a predictor of mean trough cyclosporine levels in our study (Table I). Indeed, the urinary $6-\beta-F / F F$ measurements did not at all correlate with the cyclosporine blood levels observed in the patients.

In summary, we found a significant correlation between the ERMBT result and urinary $6-\beta-\mathrm{F} / \mathrm{FF}$ ratio when all patients studied were considered. However, there were marked discrepancies between the results of the two assays in some patients, clearly indicating that the tests do not always give similar information about the status of P450IIIA catalytic activity in a patient. Of the two tests, the ERMBT would appear to provide the most relevant information for individualization of the dosing of substrates of P450IIIA. Our findings point out the importance of considering extrahepatic enzyme activity when designing noninvasive means of measuring liver oxidative metabolism.

\section{References}

1. Nebert DW, Nelson DR, Coon MJ, et al. The P450 superfamily: update on new sequences, gene mapping, and recommended nomenclature. DNA Cell Biol 1991; 10:1-14.

2. Watkins PB, Wrighton SA, Maurel P, et al. Identification of an inducible form of cytochrome P-450 in human liver. Proc Natl Acad Sci USA 1985;82:6310-4.

3. Kronbach T, Fischer V, Meyer UA. Cyclosporine metabolism in human liver: identification of a cytochrome P-450II gene family as the major cyclosporine-metabolizing enzyme explains interactions of cyclosporine with other drugs. Clin Pharmacol Ther 1988;43:630-5.

4. Kronbach T, Mathys D, Umeno M, Gonzalez FJ, Meyer UA. Oxidation of midazolam and triazolam by human liver cytochrome P450IIIA4. Mol Pharmacol 1989;36:89-96.

5. Bargetzi MJ, Aoyama T, Gonzalez FJ, Meyer UA. Li- docaine metabolism in human liver microsomes by cytochrome P450lula4. Clin Pharmacol Ther 1989;46: 521-7.

6. Jacolot F, Simon I, Dreano Y, Beaune P, Riche C, Berthou F. Identification of the cytochrome P450IIIA family as the enzymes involved in the $\mathrm{N}$-demethylation of tamoxifen in human liver microsomes. Biochem Pharmacol 1991:41:1911-9.

7. Guengerich FP, Martin MV, Beaune PH, Kremers P, Wolff T, Waxman DJ. Characterization of rat and human liver microsomal cytochrome P-450 forms involved in nifedipine oxidation, a prototype for genetic polymorphism in oxidative drug metabolism. J Biol Chem 1986;261:5051-60.

8. Shimada T, Guengerich FP. Evidence for cytochrome $\mathrm{P}-450 \mathrm{NF}$, the nifedipine oxidase, being the principal enzyme involved in the bioactivation of aflatoxins in human liver. Proc Natl Acad Sci USA 1989;86:462-5.

9. Ramsdell HS, Parkinson A, Eddy AC, Eaton DL. Bioactivation of aflatoxin $\mathrm{B}_{1}$ by human liver microsomes: role of cytochrome P450 IIIA enzymes. Toxicol Appl Pharmacol 1991;108:436-47.

10. Guengerich FP. Characterization of human microsomal cytochrome P-450 enzymes. Annu Rev Pharmacol Toxicol 1989;29:241-64.

11. Watkins PB. Role of cytochromes P450 in drug metabolism and hepatotoxicity. Semin Liver Dis 1990;10: 235-50.

12. Watkins PB, Murray SA, Winkelman LG, Heuman DM, Wrighton SA, Guzelian PS. Erythromycin breath test as an assay of glucocorticoid-inducible liver cytochromes P-450. J Clin Invest 1989;83:688-97.

13. Baker AL, Kotake AN, Schoeller DA. Clinical utility of breath tests for the assessment of hepatic function. Semin Liver Dis 1983;3:318-29.

14. Watkins PB, Hamilton TA, Annesley TM, Ellis CN, Kolars JC, Voorhees JJ. The erythromycin breath test as a predictor of cyclosporine blood levels. CiIN PHARMACOL Ther 1990;48:120-9.

15. Lown K, Kolars J, Turgeon DK, Merion R, Wrighton SA, Watkins PB. The erythromycin breath test selectively measures P450IIIA in patients with severe liver disease. Clin Pharmacol Ther 1992;52:229-38.

16. Waxman DJ, Attisano C, Guengerich FP, Lapenson DP. Human liver microsomal steroid metabolism: identification of the major microsomal steroid hormone 6 beta-hydroxylase cytochrome P-450 enzyme. Arch Biochem Biophys 1988;263:424-36.

17. Ged C, Rouillon JM, Pichard L, et al. The increase in urinary excretion of $6 \beta$-hydroxycortisol as a marker of human hepatic cytochrome P450IIIA induction. $\mathrm{Br} \mathrm{J}$ Clin Pharmacol 1989;28:373-87.

18. Bienvenu T, Rey E, Pons G, D'Athis P, Olive G. A simple non-invasive procedure for the investigation of cytochrome P-450 IIIA dependent enzymes in humans. Int $\mathbf{J}$ Clin Pharmacol Ther Toxicol 1991; $29: 441-5$. 
19. Hunt CM, Watkins PB, Saenger $P$, et al. Heterogeneity of CYP3A isoforms metabolizing erythromycin and cortisol. Clun Pharmacol Ther 1992;51:18-23.

20. Saenger P. 6-beta-hydroxycortisol in random urine samples as an indicator of enzyme induction. Clin PharmaCOL THER 1983;34:818-21.

21. Voccia E, Saenger P, Peterson RE, et al. 6- $\beta$-Hydroxycortisol excretion in hypercortisolemic states. J Clin Endocrinol Metab 1979;48:467-71.

22. Vescei P. Glucorticoids: Cortisol, corticosterone, and compound S. In: Jaffe BM, Behrman HR, eds. Methods of hormone radioimmunoassay. New York: Academic Press, 1974:393-415.

23. Annesley T, Matz K, Balogh L, Clayton L, Giacherio D. Liquid-chromatographic analysis for cyclosporine with use of a microbore column and small sample volume. Clin Chem 1986;32:1407-9.

24. Thrasher K, Werk EE Jr, Choi Y, Sholiton LJ, Meyer $W$, Olingert $C$. The measurement, excretion, and source of urinary 6-hydroxycortisol on humans. Steroids 1969:4:455-68.

25. Watkins PB, Wrighton SA, Schuetz EG, Molowa DT, Guzelian PS. Identification of glucocorticoid-inducible cytochromes P-450 in the intestinal mucosa of rats and man. J Clin Invest 1987;80:1029-36.

26. Schuetz EG, Schuetz JD, Grogan WM, et al. Expression of cytochrome P450A in amphibian, rat and human kidney. Arch Biochem Biophys 1992;294:206-14.

27. Kolars JC, Awni WM, Merion RM, Watkins PB. Firstpass metabolism of cyclosporin by the gut. Lancet 1991;338:1488-90.

28. Kolars JC, Stetson PL, Rush BD, et al. Cyclosporine metabolism by P450IIIA in rat enterocytes - another determinant of oral bioavailability? Transplantation 1992;53:596-602.

29. Kishida S, Fukushima DK. Radioimmunoassay of $6 \beta-$ hydroxycortisol in human plasma and urine. Steroids 1977;30:741-9.

30. Nahoul K, Adeline J, Paysant F, Scholler R. Radioimmunoassay of plasma and urine $6 \beta$-hydroxycortisol: levels in healthy adults and in hypercortisolemic states. J Steroid Biochem 1982:17:343-50.

31. Lipman MM, Katz FH, Jailer JW. An alternate pathway for cortisol metabolism: $6 \beta$-hydroxy-cortisol production by human tissue slices. J Clin Endocrinol 1962:22:268-72. 